\title{
Scenario Analysis on Climate Change Impacts of Urban Land Expansion under Different Urbanization Patterns: A Case Study of Wuhan Metropolitan
}

\author{
Xinli Ke, ${ }^{1}$ Feng $\mathrm{Wu}^{2}$ and Caixue $\mathrm{Ma}^{3}$ \\ ${ }^{1}$ College of Land Management, Huazhong Agricultural University, Wuhan 430079, China \\ ${ }^{2}$ State Key Laboratory of Water Environment Simulation, School of Environment, Beijing Normal University, Beijing 100875, China \\ ${ }^{3}$ College of Economics \& Management, Huazhong Agricultural University, Wuhan 430079, China \\ Correspondence should be addressed to Xinli Ke; kexl@igsnrr.ac.cn
}

Received 15 July 2013; Revised 3 October 2013; Accepted 16 October 2013

Academic Editor: Xiangzheng Deng

Copyright (C) 2013 Xinli Ke et al. This is an open access article distributed under the Creative Commons Attribution License, which permits unrestricted use, distribution, and reproduction in any medium, provided the original work is properly cited.

\begin{abstract}
Urban land expansion plays an important role in climate change. It is significant to select a reasonable urban expansion pattern to mitigate the impact of urban land expansion on the regional climate in the rapid urbanization process. In this paper, taking Wuhan metropolitan as the case study area, and three urbanization patterns scenarios are designed to simulate spatial patterns of urban land expansion in the future using the Partitioned and Asynchronous Cellular Automata Model. Then, simulation results of land use are adjusted and inputted into WRF (Weather Research and Forecast) model to simulate regional climate change. The results show that: (1) warming effect is strongest under centralized urbanization while it is on the opposite under decentralized scenario; (2) the warming effect is stronger and wider in centralized urbanization scenario than in decentralized urbanization scenario; (3) the impact trends of urban land use expansion on precipitation are basically the same under different scenarios; (4) and spatial distribution of rainfall was more concentrated under centralized urbanization scenario, and there is a rainfall center of wider scope, greater intensity. Accordingly, it can be concluded that decentralized urbanization is a reasonable urbanization pattern to mitigate climate change in rapid urbanization period.
\end{abstract}

\section{Introduction}

Urbanization is one of the most important anthropogenic influences on climate. By transforming the nature land cover to the artificial state, and anthropogenic heat emissions, the process of urbanization has a profound impact on global climate change [1]. Therefore, with the rapid urbanization, the impact of urbanization especially urban land expansion on global climate change has been one of the hotspots in research fields [2-5]. Using 40 years' climate change data and land use data of Hong Kong, Ka and Edward (2013) found that with the evolution of natural vegetation and rural landscape to urban landscape, the trend of rising temperatures in urban areas has become more apparent [6]. Combined with remote sensing and spatial statistical model, Xiong et al. (2012) studied the impact of four periods of urban land expansion on surface temperature from 1990 to 2009 in Guangzhou, and found that there is a significant correlation between the urban heat island intensity and urban land expansion in Guangzhou City [7]. Guo et al. (2012) constructed urbanization index according to different land covers and analyzed effects of different degrees of urbanizations on surface temperature [8]. The results indicated that urbanization and urban surface temperature index had a complex relationship. Kishtawal et al. (2010) assessed effects of urbanization on Indian summer heavy rain [9]. Results showed that torrential rain is more likely to occur in rapid urbanization areas than other areas. Numerous studies have demonstrated that rapid urbanization, especially urban land expansion significantly affects regional temperature and precipitation.

China has reached the "urban tipping point," with about $52.57 \%$ percent of its population now living in cities. By 2030 , the number of people who live in cities will likely be one billion. Nowadays, urban agglomeration has become 
the main feature of China's urbanization. A large body of the literature studied the effect of city size on economic efficiency while few researches pay attention to figure out climate change effects of different urbanization patterns, especially spatial patterns of urban sprawling. Chen et al. (2013) analyzed relationship between urbanization and economic growth in China and found that China's urbanization process has progressed faster than economic growth since 2004, so it is the right time that China should rethink underurbanization and its countermeasure in development strategy [10]. Salvati et al. (2013) discovered the transition from compact towards dispersed urban form and the consequent changes in Land Cover Relationships observed from 1960 to 2009 in Attica (Greece) [11]. Based on historical land use data, Wu et al. (2011) characterized the temporal patterns of Phoenix and Las Vegas using landscape pattern metrics at multiple spatial resolutions [12]. However, few studies have focused on different impacts of city size and different patterns of urban land expansion on climate change, especially climate effects of urban land expansion in urban agglomerations.

Wuhan Metropolitan is the comprehensive reform pilot area of resource-saving and environment-friendly society which Chinese government approved. It is the emerging urban cluster in China following the Bohai Rim, the Yangtze River Delta, and Pearl River Delta. In recent years, both economic development and urban land expansion in Wuhan Metropolitan are rapid. Meanwhile, the Wuhan Metropolitan urban land expansion has a significant impact on regional climate [13]. Therefore, it is urgent to figure out the impact of different urban land expansion patterns on climate change in Wuhan Metropolitan, that will find out a reasonable urban land expansion pattern to mitigate regional climate change in Wuhan Metropolitan.

This paper set up three kinds of urbanization scenarios: baseline scenario, centralized urban land expansion scenario, and decentralized urban land expansion scenario. Then, the Partitioned and Asynchronous Cellular Automaton Model is employed to simulate urban land expansion patterns under different scenarios in Wuhan Metropolitan in 2020. The results are taken as a land use/land cover underlying surface data, and input it into the WRF model, and simulated impacts of urban land expansion on regional climate change under three different scenarios of urban land expansion pattern. Through comparative analysis of three different scenarios of urban land expansion in the temporal and spatial impacts of climate change, refining to get a reasonable response of urbanization patterns to climate change in Wuhan Metropoli$\tan$.

\section{Study Area and Data Sources}

2.1. Study Area. Wuhan Metropolitan is located in the middle of Hubei province, China. It is the urban agglomeration which takes Wuhan as the center, and is composed of 8 cities of Huangshi, Ezhou, Huanggang, Xiaogan, Xianning, Xiantao, Tianmen, and Qianjiang within $100 \mathrm{~km}$, with a total area of about $5.78 \times 10^{4} \mathrm{~km}^{2}$, accounting for $31.1 \%$ of land area in Hubei Province (Figure 1). By the end of

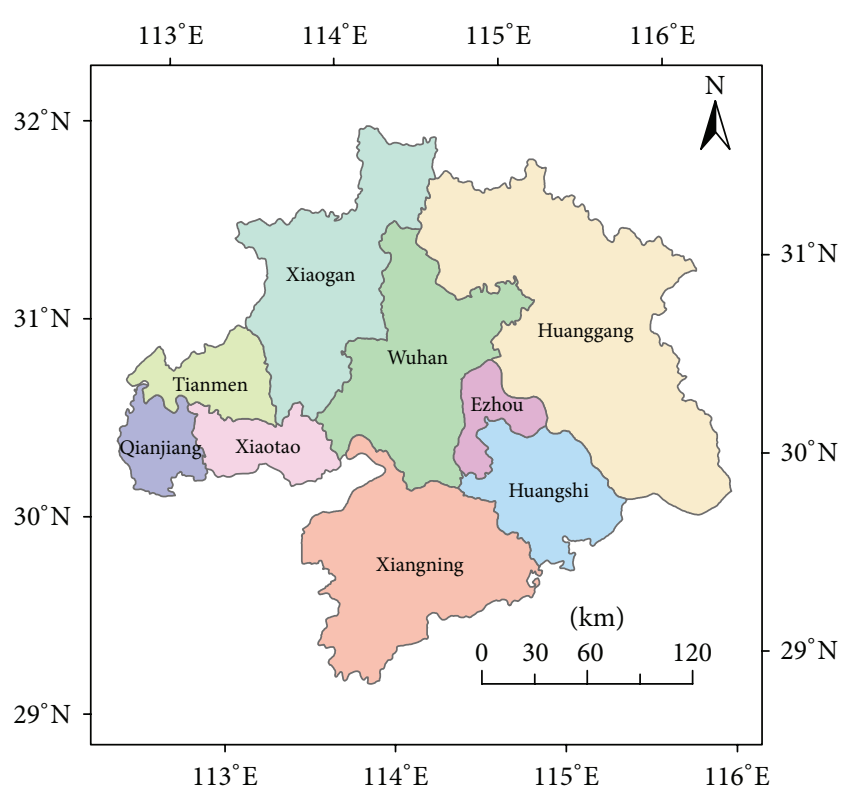

FIGURE 1: Study area.

2008, the resident population of Wuhan Metropolitan was 2994.6 million, the annual GDP reached 697.211 billion Yuan, accounting for $61.5 \%$ of the province's total GDP; it is also an important economic development center in Hubei Province, and an important strategic fulcrum of "rise of central China." Wuhan Metropolitan faces many development opportunities as the comprehensive reform pilot area of "resource-saving and environment-friendly" society. However, as China's first "resource-saving and environmentfriendly" society construction pilot area, it is necessary for Wuhan Metropolitan to consider the rapid development of not only socioeconomic development but also concerned about the ecological and environmental effects in the fast urbanization process. Therefore, it is beneficial to select Wuhan Metropolitan reasonable urbanization patterns analyzing effects of different urbanization pattern on regional climate from the perspective of climate change. It is of great significance for Wuhan Metropolitan "resource-saving and environment-friendly" society construction and it provides a new perspective for the urban land expansion chosen.

2.2. Data Sources. The data of this paper included Wuhan Metropolitan's land use data, natural environment condition data, and social-economy statistical data.

2.2.1. Land Use Data. Land use data is mainly used for scenario simulations of land use change, including two remote sensing image interpretation data of Wuhan Metropolitan in 2000 and 2008, covering five land types (farmland, forestland, grassland, built-up land, and water bodies). Among them, land use data in 2000 came from the Land Use Database of Data Center Resources and Environment, Chinese Academy of Science [14]. The resource of this database is Landsat $\mathrm{TM} / \mathrm{ETM}^{+}$image interpretation with a spatial resolution of 
the $30 \mathrm{~m} \times 30 \mathrm{~m}$. And then, the land use dataset is resampled to $100 \mathrm{~m} \times 100 \mathrm{~m}$ raster dataset. Land use data in 2008 came from the CBERS (China-Brazil Earth Resource Satellite) image interpretation with a spatial resolution of $20 \mathrm{~m} \times$ $20 \mathrm{~m}$. The results of remote sensing interpretation are also resampled to $100 \mathrm{~m} \times 100 \mathrm{~m}$ grid. Specific process is as follows: using remote sensing data processing software ENVI to make geometric correction, radiometric correction, cropping boundary, supervised classification and visual interpretation, accuracy test for remote sensing image, and then generate land use data. Land use types include five land types such as farmland, forestland, grassland, built-up land, and water bodies. Built-up land covers urban land, rural residential, and other construction land.

2.2.2. Natural Environmental Conditions Data. The natural environmental conditions data involved in this study include DEM data of the study area, distance from the city at all levels, distance from the railway, distance from the road, and distance from the river. DEM data in this research came from 1:250000 DEM data of the State Bureau of Surveying and Mapping (SBSM). This paper hierarchically calculated the distance from the city at all levels to each $100 \mathrm{~m} \times 100 \mathrm{~m}$ grid. Using the Landsat TM/ETM ${ }^{+}$geometric correction in 2000 that covered Wuhan Metropolitan, laid out the major river systems and the lines of communication of the study area, worked out the distance from each $100 \mathrm{~m} \times 100 \mathrm{~m}$ grid to rail, road, and river.

2.2.3. Social-Economy Statistical Data. The social and economic statistical data in this paper include population data of Wuhan Metropolitan cities, per capita retail sales of social consumer goods, the total investment in fixed assets, per capita income, and second industry gross grain yield per unit area from 2000 to 2008. This kind of data comes from Hubei Statistical Yearbook.

2.2.4. Climate Data. Climate data includes annual precipitation data and annual average temperature data. Based on the China weather site observation data, spline interpolation arithmetic method is employed to interpolate meteorological site's observation data to get grid form of annual precipitation data and annual average temperature data in research region.

\section{Methods and Models}

3.1. Research Framework. The basic idea of scenario analysis on the impacts of urban land expansion on climate change is performed by Figure 2. Based on setting urban land expansion scenarios in Wuhan Metropolitan, the Partitioned and Asynchronous Cellular Automata Model is employed to carry out the scenarios simulation of urban land use expansion in Wuhan Metropolitan, so as to get temporal pattern of urban land expansion about the future. By converting urban land expansion simulation results to land use/land cover underlying surface data and inputting it into WRF model, regional climate change under different scenarios in Wuhan Metropolitan can be carried out. By comparing analysis on climate change effects of urban land expansion under different scenarios, it is helpful to get reasonable urban land expansion pattern for mitigation climate change in Wuhan Metropolitan.

(1) Urban Land Expansion Scenario Simulation. Urban land expansion pattern scenarios of Wuhan Metropolitan are set up firstly. In this paper, three urbanization pattern scenarios are set up. They are baseline scenario, centralized urban land expansion scenario, and decentralized urban expansion scenario. On this basis, the Partitioned \& Asynchronous Cellular Automata model is employed to simulate urban land expansion under different scenarios. In Partitioned \& Asynchronous Cellular Automata model, urban land expansion scenarios and regional socioeconomic development conditions are used to calibrate asynchronous evolving speed, regional land use change laws and regional differences of socioeconomic conditions are used to calibrate transformation rules in each partition, and built-up demand decided by economic development and farmland demand decided by food security are used to calibrate global stopping condition. After these, urban land expansion patterns under different scenarios in Wuhan Metropolitan can be carried out.

(2) Scenario Analysis of Urban Land Expansion on Climate Change. The underlying surface data of Wuhan Metropolitan for climate model are processed firstly. By scale transformation and type transformation, Scenario simulation results of urban land expansion can be converted to underlying surface data for WRF model. Then, keeping lateral boundary conditions and all other parameters of WRF model constant, processed Land Use/Land Cover underlying surface data are input into WRF model to simulate regional climate change in Wuhan Metropolitan. After these processes, regional climate change under different urbanization patterns in Wuhan Metropolitan can be figured out by WRF model. Through scenario analysis of regional climate change under different urbanization patterns, reasonable urbanization patterns which can mitigate climate change in Wuhan Metropolitan can be worked out.

\subsection{Partitioned and Asynchronous Cellular Automata Model.} Cellular automata model has the ability of simulating spatial and temporal evolution in complex systems. Their "bottomup" research idea fully reflects the concept that the local individual behavior of complex systems produces global and orderly pattern. Therefore, cellular automata model has natural advantages in urban land expansion simulation [15]. However, most cellular automat models have some limitations in simulating urban land expansion. On the one hand, it ignores spatial heterogeneities existing in urban land expansion and its influencing factors to use the unified cellular transformations rules for all cellular in urban land expansion simulation. On the other hand, it ignores the spatial heterogeneities of urban land expansion speed to employ same evolving speed for all cellular. Both of them become the barrier of simulation accuracy improving for cellular automata model [16]. To solve this problem, Ke (2010) proposed a Partitioned \& Asynchronous Cellular 


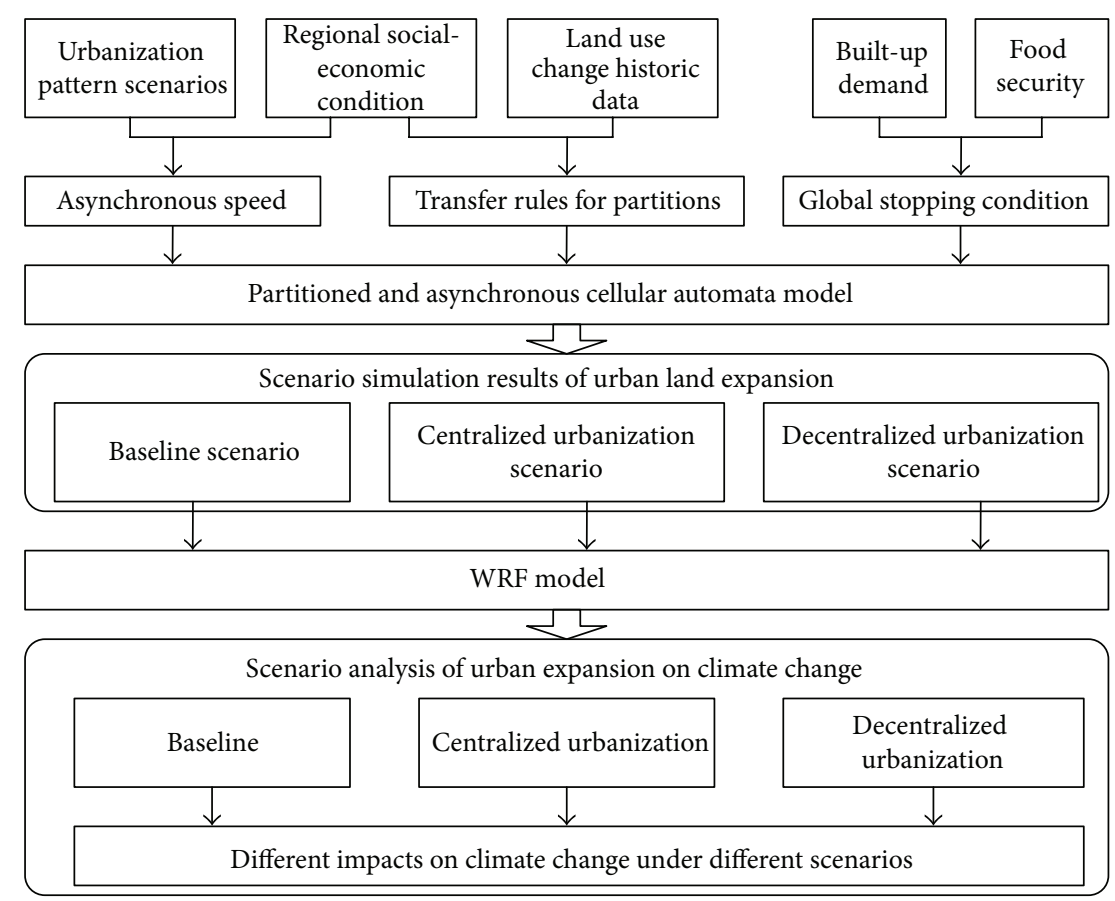

FIGURE 2: Research framework of scenario analysis of urban land expansion on climate change impacts.

Automata Model based on spatial data mining [17]. In this model, spatial data mining methods are employed to dig out partitions for cellular automata model and separately cellular transformation rules for each partition are dug out by Decision Tree Algorithm. On the other hand, evolving speed for each cellular is calculated grid by grid. Combining with transformer rules for each partition and evolving speed for each cellular, more accurate simulation result of urban land expansion can be worked out.

Transformation rules for each partition are made up of three sections: transformation probability for each partition, unit constraints, and neighborhood development density [15]. It can be showed by the following formula:

$$
P_{d, i j}^{t}=\left[1+(-\ln \gamma)^{\alpha}\right] \times P_{g} \times \operatorname{con}\left(s_{i j}^{t}\right) \times \Omega_{i j}^{t},
$$

where $p_{d, i j}^{t}$ is transformation probability for each partition, $\gamma$ is random number ranging from 0 to 1 , and $\alpha$ is the parameter which is controlling random variable effect level. It is an integer which ranges from 1 to $10, P_{g}$ is transformation probability which is decided by urban expansion influencing factors, $\operatorname{con}\left(s_{i j}^{t}\right)$ is constraint condition of unit, $\Omega_{i j}^{t}$ is neighborhood function which means effect of neighborhood on cellular automata transformation probability.

In the above parameters, $\gamma$ and $\alpha$ are introduced to add random factors in cellular automata model, using to imitate effect and intervention of all kinds of uncertain factors in the process of land use. $P_{g}$ is obtained from the geographical phenomenon change data and related impact factors by the method of spatial data mining. It remains unchanged in the process of the whole simulation.
$\Omega_{i j}^{t}$ is a very important factor. It changes over time and can be performed by the following formula:

$$
\Omega_{i j}^{t}=\frac{\sum_{3 \times 3}\left(s_{i j}=\text { target }\right)}{3 \times 3-1},
$$

where $\operatorname{con}\left(s_{i j}^{t}\right)$ is unit constraint condition representing cellular which cannot transform to urban land, such as water and high mountain. For example, $\operatorname{con}\left(s_{i j}^{t}\right)=0$, where cellular represent basic farmland preservation area, because it is constraint to development in this area.

$P_{g}$ is regional land use change regulations mined from data of the regional land use and related influencing factors. In our research, C5.0 Decision Tree Algorithm is employed to calculated regional land use change rules. Decision Tree Algorithm is a typical data mining classification algorithm. Its main role is to reveal the structured information in the data. The created tree structure is visual, easy to understand, and easy to deal with describing data of nonlinear data, the hidden knowledge rules in data can be extracted. Therefore, the Decision Tree Algorithm can be used to dig out cellular transformation rule in cellular automata [17]. In C5.0 Tree Decision Algorithm, clusters are decided by information gain which can be calculated by the following formula:

$$
I\left(r_{1}, r_{2}, \ldots, r_{m}\right)=-\sum_{i=1}^{m} p_{i} \cdot \log _{2}\left(p_{i}\right),
$$

where, $r_{i}$ is the subset of dataset $S$ which belongs to cluster $C_{i}$, $p_{i}$ is the probability of every sample that belongs to $C_{i}, I$ is the information gain. 
Asynchronous evolving speed is determined by two parts, level of social-economic development and urbanization scenario. Compared with the conditions of social and economic development level, urbanization patterns play much more important role in urban land expansion speed. Therefore, in order to clarify the influential difference of cities urban land expansion on regional climate change in different urbanization pattern of Wuhan Metropolitan, asynchronous evolving speed for Partitioned \& Asynchronous Cellular Automata Model is decided by urbanization patterns. In baseline scenario, the evolving speed for each cellular in Wuhan Metropolitan follows in the history of law, mainly determined by the regional differences of socio-economic development. In centralized urbanization scenario, the greater the cities are, the higher the urban land expansion prefers and the faster the urban land expand. In decentralized urbanization scenario, smaller the size of cities is, the higher priority and slower the speed of urban land expansion are. Asynchronous evolving speed in Partitioned \& Asynchronous Cellular Automata Model can be figured out by the following formula:

$$
v_{i j_{\text {_pri }}}=\frac{\text { priority }_{i j}}{\text { priority }_{\max }-\text { priority }_{\min }} \times\left(v_{\max }-v_{\min }\right)+v_{\min },
$$

where, $v_{i j}$ is transformation speed of cell $(i, j)$, priority is $_{i j}$ urban land development priority of cell $(i, j)$, priority max $_{\text {max }}$ is the maximum of all-region's urban land development priority, priority $\min _{\min }$ is the minimum of all-region's urban land development priority, $v_{\max }$ and $v_{\min }$ are all-region's maximum and minimum of cellular transformation speed.

Since cellular automaton model does not have the concept of transform speed but only evolution interval, so we need to convert evolutionary speed to evolution interval here as follows:

$$
\text { Interval }_{i j \_ \text {pri }}=\left[\frac{1}{v_{i j \_p r i}}\right]
$$

The above formula is the corresponding evolution interval of evolving speed. Accordingly, we can estimate asynchronous evolution interval of urban land expansion in Wuhan Metropolitan.

3.3. WRF Model. WRF model is a new generation mesoscale weather forecasting models which were jointly initiated by the U.S. Center for Atmospheric Research in middle and small scale meteorological department, the National Centers for Environmental Prediction Environmental Simulation Center, Forecasting system forecast research lab, and the storm prediction center of Oklahoma University. The key consideration of WRF model is forecast of important weather process from the cloud scale to the synoptic scale, including preprocessing module WPS (WRF processing system) and main module ARW. WPS is not only the preprocessing part of mode data, but also the part that provides some initial boundary before the three-dimensional variation systems established. It is mainly responsible for the standard grid data preprocessing and terrain data preprocessing. WPS modules include three sub-modules: geogrid, ungrib, and metgrid. Among them, main function of geogrid submodule is to define and create areas of patterns. In the geogrid module, users can set the projection of the simulation domain, range size, regional location, nesting, and other parameters. According to these custom settings, geogrid will interpolate topography, land use, soil type, and other data to the defined region network, the data format is NetCDF. Ungrib submodule's main function is converting standard grib format data files into a format that can be recognized by metgrid. Typically grib data files have many different formats; the same meteorological elements may have different elements code. For these different formats, WPS provides the corresponding Vtable function pointer, such as AWIP, GFS, and so forth. Metgrid sub-module's main function is meteorological data interpolation. It interpolates the meteorological of large area into calculated grid of pattern (including the horizontal direction and the vertical direction), and provides initial and boundary condition file for the model.

In WRF model, the original land use data comes from the global 24 types of land use types of United States Geographical Survey land use systems (US Geological Survey land use, USGS). Each land-use types have different roughness, albedo, and other parameters, affecting the flow of meteorological fields, precipitation, temperature, or temperature.

In this study, the urban land expansion scenario simulation results in Wuhan Metropolitan were spatially resampled. We substituted urban land use of simulation results for corresponding land use type in the original land use data in WRF model to format new underlying surface data. So it could be used to simulate urban land expansion climatic effects.

\section{Results}

4.1. Urbanization Pattern Scenarios for Wuhan Metropolitan. Urbanization patterns are divided into two categories: centralized and decentralized urbanization. Centralized urbanization has three main features. First, the urban scale structure is centralized. It is mainly performed that large cities, metropolitan and medium-sized cities lead in development. Second, cluster is the main feature of city metropolitan. It is mainly showed that metropolitans in various grades are developing rapidly. Third, urban land use becomes more intensified spatially. In our research, centralized urbanization mainly refers to the first characteristic, namely, cities, metropolitan, and medium-sized cities that lead in development. Corresponding decentralized urbanization, the main characteristic is that small towns lead in development.

According to urbanization patterns, three scenarios of urban land expansion are designed. There are baseline scenario, centralized urban land expansion scenario, and decentralized urban land expansion scenario in this research. Under the baseline scenario, urban land expansion in Wuhan Metropolitan follows its historical law. In centralized urban land expansion scenario, large cities lead in development, while small towns of urban land use expansion speed 
TABLE 1: Asynchronous evolution interval of urban land expansion in Wuhan Metropolitan under different scenarios.

\begin{tabular}{|c|c|c|c|}
\hline \multirow{2}{*}{ Name } & \multicolumn{3}{|c|}{ Urban land expansion scenarios } \\
\hline & Baseline & Centralized & Decentralized \\
\hline Wuhan & 1 & 1 & 20 \\
\hline Caidian & 7 & 5 & 15 \\
\hline Jiangxia & 7 & 5 & 15 \\
\hline Huangpi & 4 & 5 & 15 \\
\hline Xizhou & 4 & 5 & 15 \\
\hline Ezhou & 4 & 10 & 10 \\
\hline Huangshi & 2 & 10 & 10 \\
\hline Daye & 5 & 20 & 1 \\
\hline Yangxin & 4 & 20 & 1 \\
\hline Huanggang & 4 & 10 & 10 \\
\hline Macheng & 6 & 20 & 1 \\
\hline Wuxue & 4 & 20 & 1 \\
\hline Tuanfeng & 6 & 20 & 1 \\
\hline Hong'an & 6 & 20 & 1 \\
\hline Luotian & 4 & 20 & 1 \\
\hline Yingshan & 2 & 20 & 1 \\
\hline Xishui & 4 & 20 & 1 \\
\hline Qichun & 4 & 20 & 1 \\
\hline Huangmei & 5 & 20 & 1 \\
\hline Xiaogan & 3 & 10 & 10 \\
\hline Yingcheng & 5 & 20 & 1 \\
\hline Anlu & 5 & 20 & 1 \\
\hline Hanchuan & 4 & 20 & 1 \\
\hline Dawu & 5 & 20 & 1 \\
\hline Yunmeng & 4 & 20 & 1 \\
\hline Xianning & 8 & 10 & 10 \\
\hline Chibi & 5 & 20 & 1 \\
\hline Jiayu & 5 & 20 & 1 \\
\hline Tongcheng & 3 & 20 & 1 \\
\hline Chongyang & 3 & 20 & 1 \\
\hline Tongshan & 3 & 20 & 1 \\
\hline Xiantiao & 5 & 20 & 1 \\
\hline Tianmen & 5 & 20 & 1 \\
\hline Qianjiang & 7 & 20 & 1 \\
\hline
\end{tabular}

is controlled. In decentralized urban land expansion scenario, priority in the development is achieved by small towns, metropolitan urban land expansion are controlled. Accordingly, by setting the asynchronous evolution interval of Partitioned \& Asynchronous Cellular Automata Model, urban land expansion patterns in Wuhan Metropolitan under different scenarios can be simulated. Taking centralized urbanization scenario for example, large cities will receive the prior development. At the same time, cities have higher evolving speed, that evolution should be given a smaller interval. Accordingly, we could set three asynchronous evolution intervals of urban land expansion under different scenarios in Wuhan Metropolitan (Table 1). In each scenario, the demand of urban land in whole Wuhan Metropolitan is $819784 \mathrm{hm}^{2}$, while the demand of farmland in the whole is $2574194 \mathrm{hm}^{2}$.
4.2. Scenario Simulation of Urban Land Expansion of Wuhan Metropolitan. According to asynchronous evolution intervals scenarios set by urban land expansion scenario in Wuhan Metropolitan, combing transform rules for each partition and global stopping conditions which were determined by socioeconomic conditions and natural resource endowments in Wuhan Metropolitan, the Partitioned \& Asynchronous Cellular Automata Model can be employed to carry out urban land expansion patterns under each scenario in Wuhan Metropolitan (Figure 3). As could be seen, spatial patterns of urban land use results in Wuhan Metropolitan which are very similar under the baseline scenario and the centralized scenario. The difference lies in baseline scenario, the speed of urban land expansion in big cities and small cities had some difference, but both developed; while in centralized urbanization scenario, big cities and small cities' speed of urban land use expansion has a very significant difference. There were very rapid urban land use expansion in Wuhan and its surrounding areas, but urban land use of other areas in Wuhan Metropolitan expanded very slowly. Compared with former two scenarios, urban land use expansion law of Wuhan Metropolitan is distinctly different in decentralized urbanization scenario: Wuhan and its surrounding areas of urban land expansion are very small, while the urban land expansion is obvious in corresponding small urban areas.

The underlying surface of WRF model is classified based on classification criteria of USGS data, while Land Use/Land Cover in urban land expansion simulation was divided into five categories: farmland, forestland, grassland, water bodies, and built-up land. Therefore, the simulation results cannot be directly used as underlying surface data to input into the WRF model. Based on original underlying surface data of WRF model, urban land expansion simulation results are converted raster datasets which have same scale with underlying surface data of WRF model by spatial analysis tools in ArcGIS10. Then, grid data in original underlying surface data are displaced by urban land use type in corresponding grid in processed urban land expansion simulation results and obtained new underlying surface data contain urban land expansion in Wuhan Metropolitan under various scenarios for WRF model (Figure 4).

4.3. Scenario Analysis of Urban Land Expansion on Climate Change Impacts. Keeping boundary conditions and other initial field of WRF model constant, substituted Wuhan Metropolitan's underlying surface data under different scenarios for WRF model. Then, regional climate change under different scenarios in Wuhan Metropolitan can be figured out by WRF model, especially spatial pattern of temperature and precipitation in Wuhan Metropolitan.

Under different scenarios of urbanization, the impact of underlying surface changes on temperature had obvious differences (Figure 5). Overall, the warming effect of the three scenarios is significantly different. Among the three scenarios, the most obvious warming effect is centralized urban land expansion scenario, followed by the baseline scenario, while the smallest warming effect is decentralized urban land expansion scenario. From the aspect of time series, the 


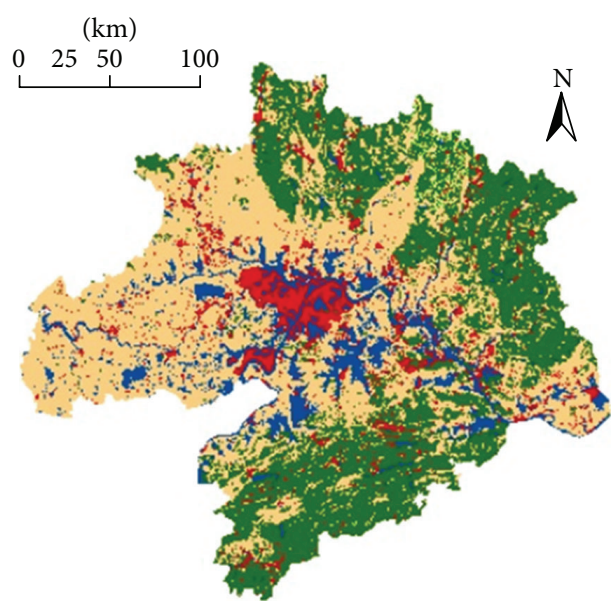

(a) Baseline scenario

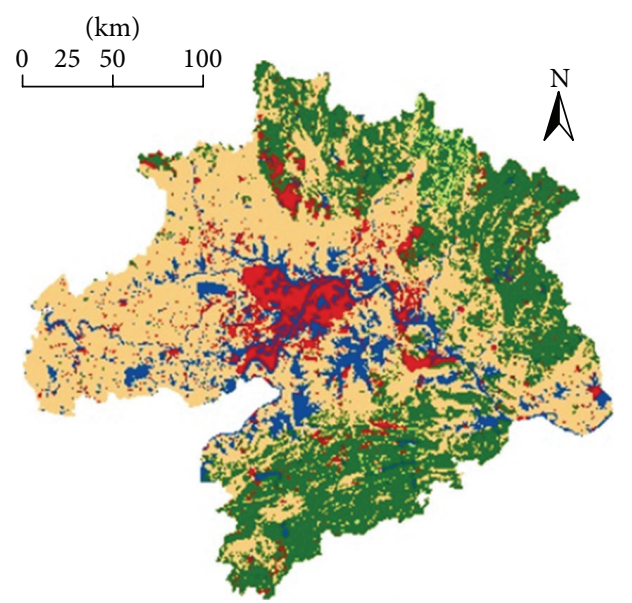

(b) Centralized urbanization scenario

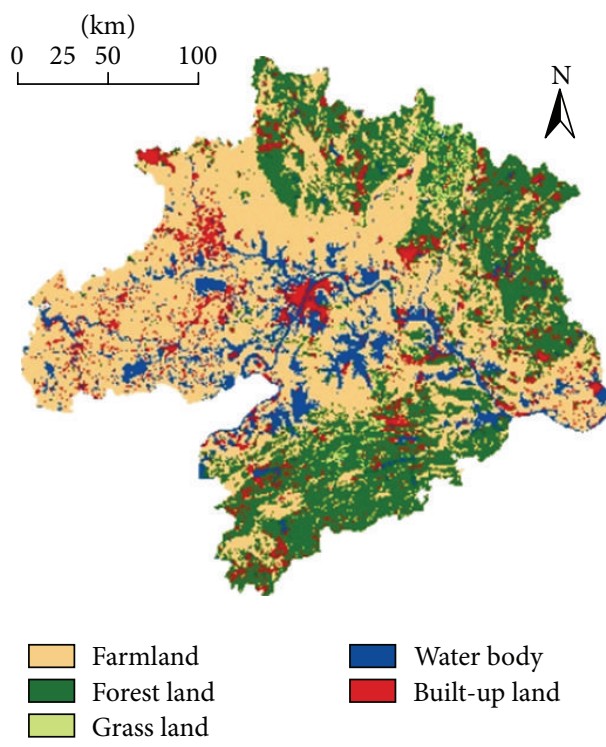

(c) Decentralized urbanization scenario

Figure 3: Spatial Pattern of Land Use under Different Urbanization Scenarios in Wuhan Metropolitan.

strongest warming effect difference of three scenarios appears at the hottest month of the year (June, July, and August). In the colder months (January, February, November, and December), the warming effect difference of three scenarios is small.

Under different scenarios of urbanization, there are significant differences of spatial patterns of impacts of land surface on temperature change (Figure 6). Seeing from differences of the three scenarios, difference between centralized urban land expansion scenario and decentralized urban land expansion scenario is the biggest, while there are small differences between centralized urban land expansion scenario and baseline scenario. Compared to decentralized urban land expansion scenario, the spatial range of warming effect is much stronger in centralized urban land expansion scenario, and the magnitude of temperature difference was greater. Compared with the baseline scenario, the spatial range of warming effect was a little stronger and more concentrated in centralized urban land expansion scenario, and the magnitude of temperature difference is also smaller. From the aspects of time series, colder month (in January as an example) warming differences are small, but the hotter months (in July for example) warming differences are much significant. From the annual average level, the biggest warming difference still lies between in centralized urban land expansion scenario and decentralized urban land expansion scenario, the minimum warming difference lies between centralized urban land expansion scenario and the baseline scenario. As could be seen, among three scenarios, the warming effect caused by underlying surface changes is widest and strongest under the centralized urban land expansion scenario. In decentralized urban land expansion scenario, the warming scope caused by the underlying surface changes is the smallest and so is magnitude. 


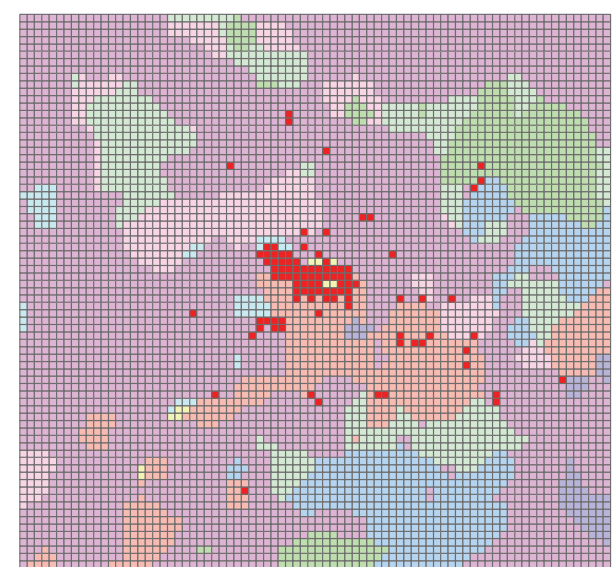

(a) Baseline scenario

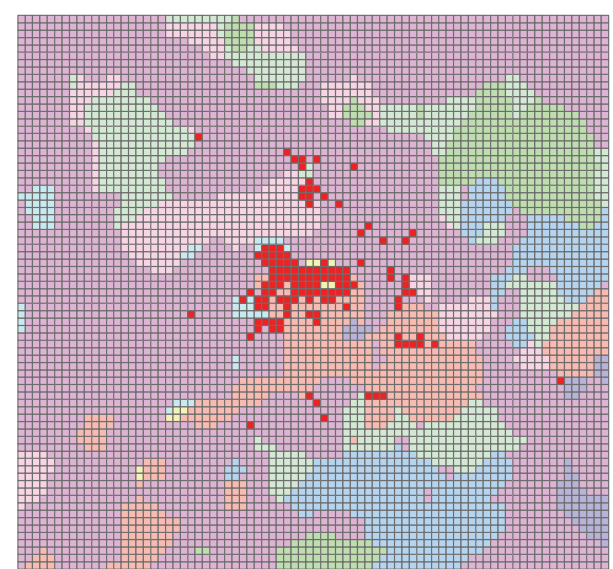

(b) Centralized urbanization scenario

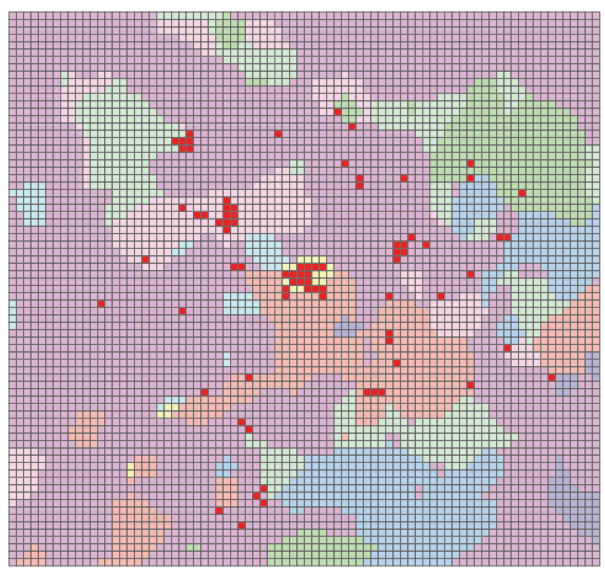

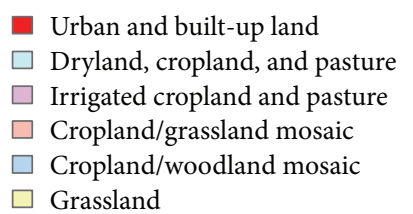
$\square$ Dryland, cropland, and pasture
$\square$ Irrigated cropland and pasture
$\square$ Grassland

$\square$ Shrubland

$\square$ Savanna

$\square$ Deciduous broadleaf forest

$\square$ Water bodies

(c) Decentralized urbanization scenario

FIgURE 4: Underlying surface data of Wuhan Metropolitan under different scenarios.

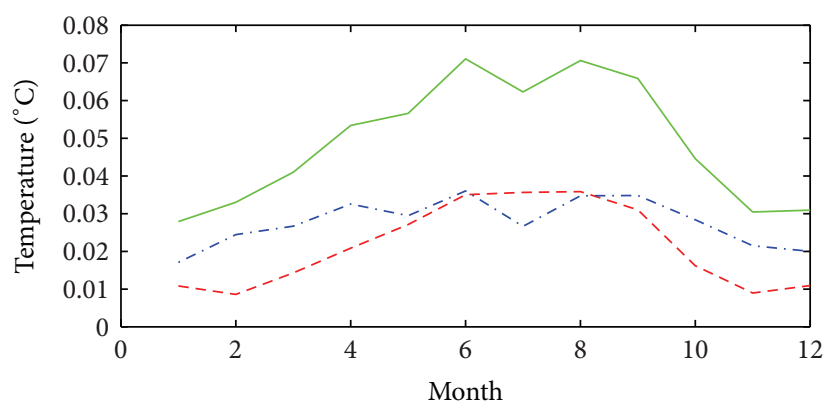

- - - Centralized-baseline

- Centralized-decentralized

. . - Baseline-decentralized

FIGURE 5: Temperature difference under different scenarios. 


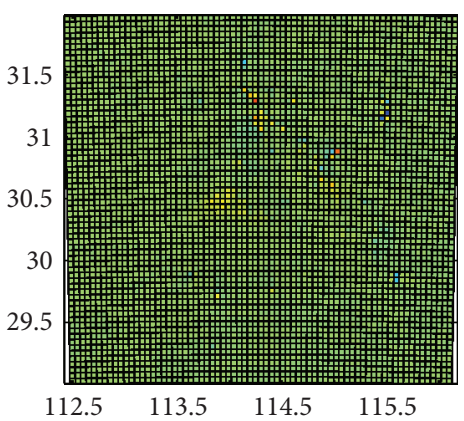

(a) January (Centralized-Baseline)

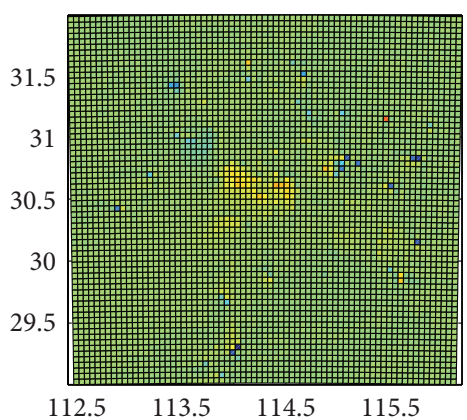

(d) January (Baseline-Decentralized)

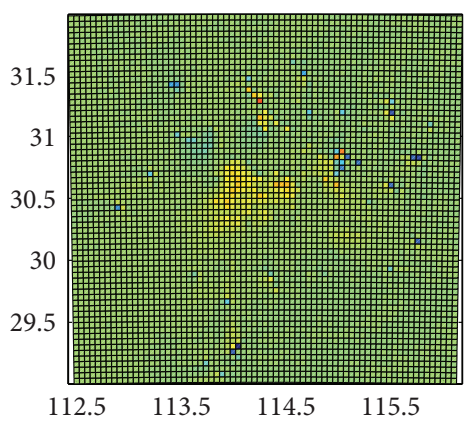

(g) January (Centralized-Decentralized)

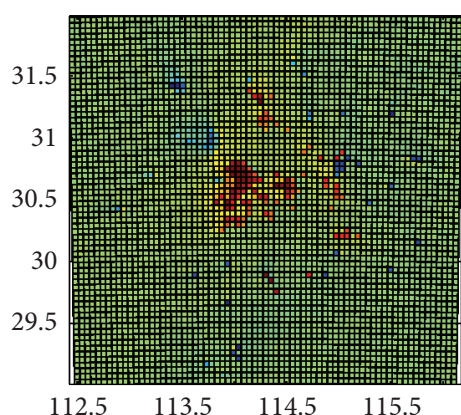

(b) July (Centralized-Baseline)

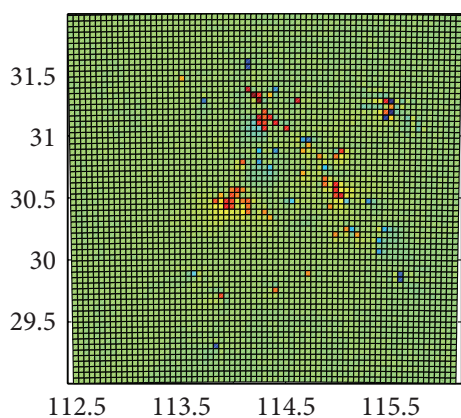

(e) July (Baseline-Decentralized)

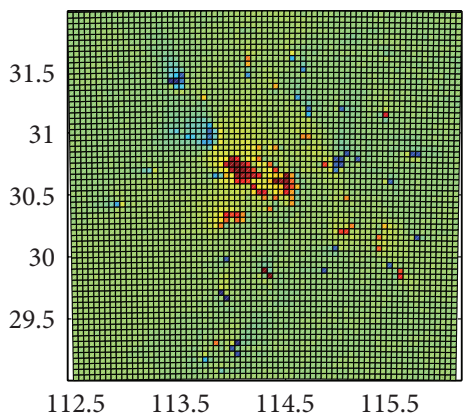

(h) July (Centralized-Decentralized)

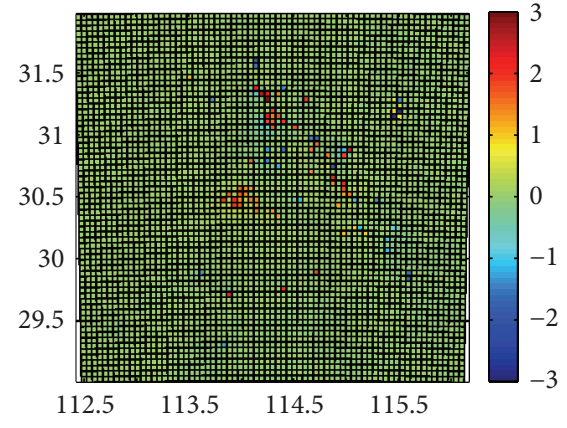

(c) Annual average (Centralized-Baseline)

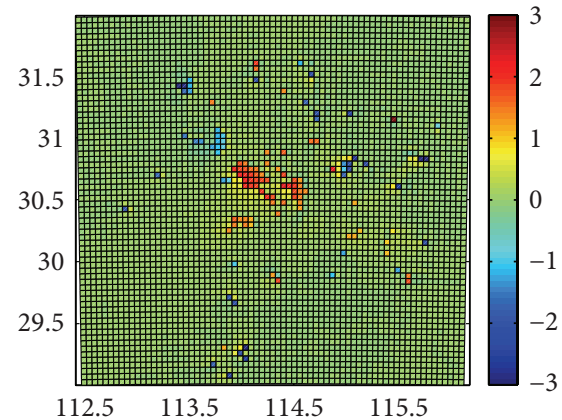

(f) Annual average (Baseline-Decentralized)

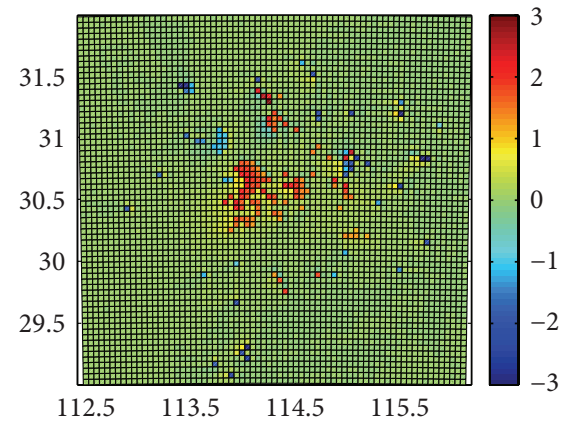

(i) Annual average (Centralized-Decentralized)

Figure 6: Spatial pattern of warming effect under different scenarios (unit: ${ }^{\circ} \mathrm{C}$ ).

Under different scenarios of urban land expansion, the difference of impact of underlying surface changes on precipitation is not particularly significant (Figure 7). Overall, the precipitation of decentralized urban land expansion scenarios is maximum, slightly higher than the other two scenarios. The precipitation of centralized urbanization scenarios is minimal, slightly lower than the other two scenarios. From the aspects of time series, precipitation under different scenarios presents an appearance of consistent in monthly change, the peak of precipitation appeared in July. Precipitation differences of three scenarios are maximum near the peak precipitation.

As the spatial distribution is concerned, precipitation of the centralized urban land expansion scenario and the baseline scenario is relatively concentrated, while spatial precipitation of the decentralized urban land expansion scenario is relatively dispersed (Figure 8 ). There is a wider scope and greater intensity of rainfall centers under centralized urban land expansion scenario and baseline scenario. In the decentralized urban land expansion scenario, precipitation center presented decentralized characteristic, there were two smaller and weaker precipitation centers which are more evenly distributed in space.

\section{Conclusions and Discussions}

Rational choice of urbanization patterns is the hot issue in government and academy society. Many economists have conducted comparative study of urbanization pattern from the perspective of economic efficiency, and given the basis of urbanization model selection from an economics. On the other hand, natural scientists have proved that urban land expansion is one of the most important reasons for global climate change. Urban land expansion has a nonnegligible 


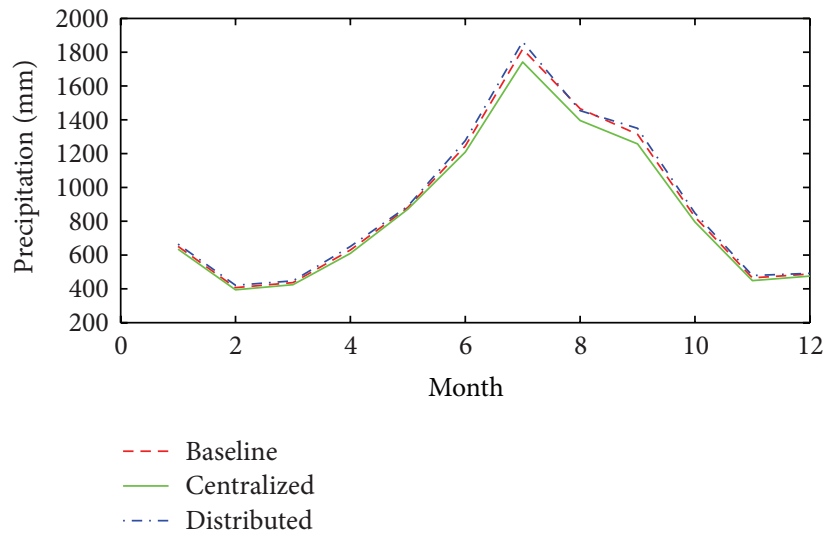

Figure 7: Precipitation under different scenarios.

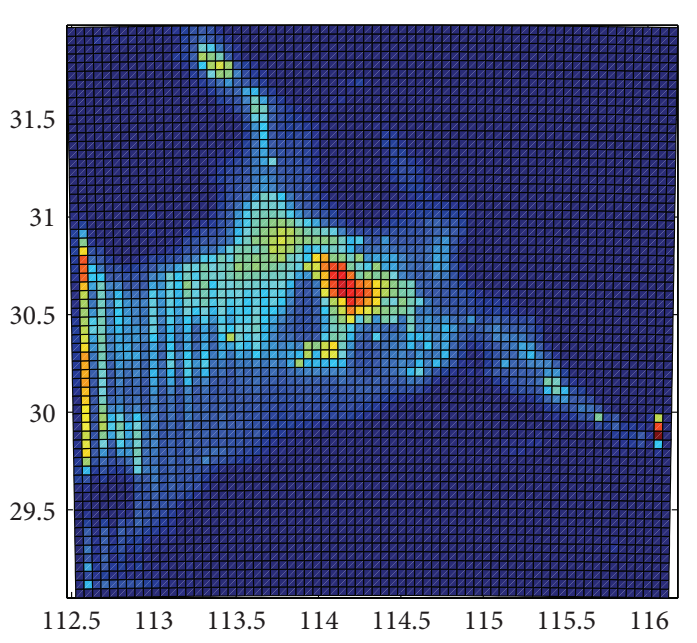

(a) Baseline scenario

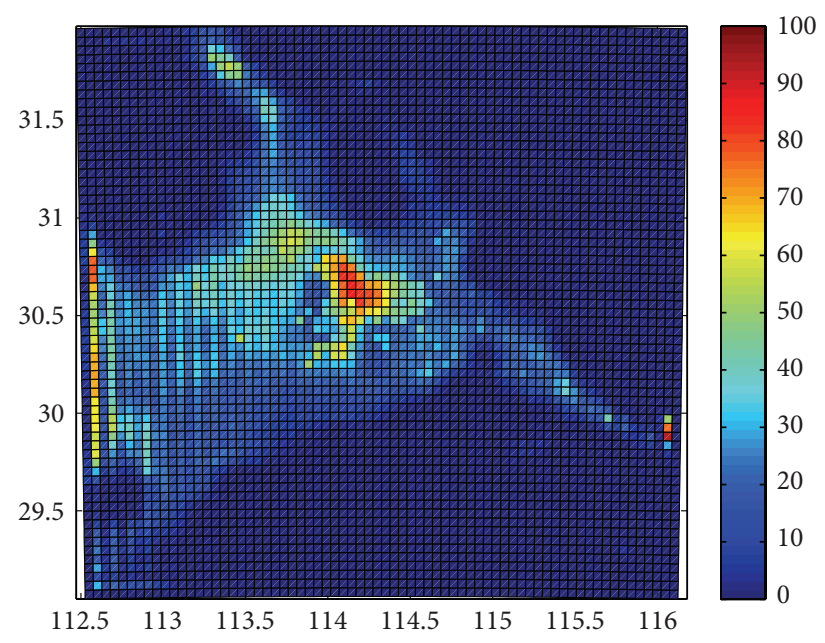

(b) Centralized scenario

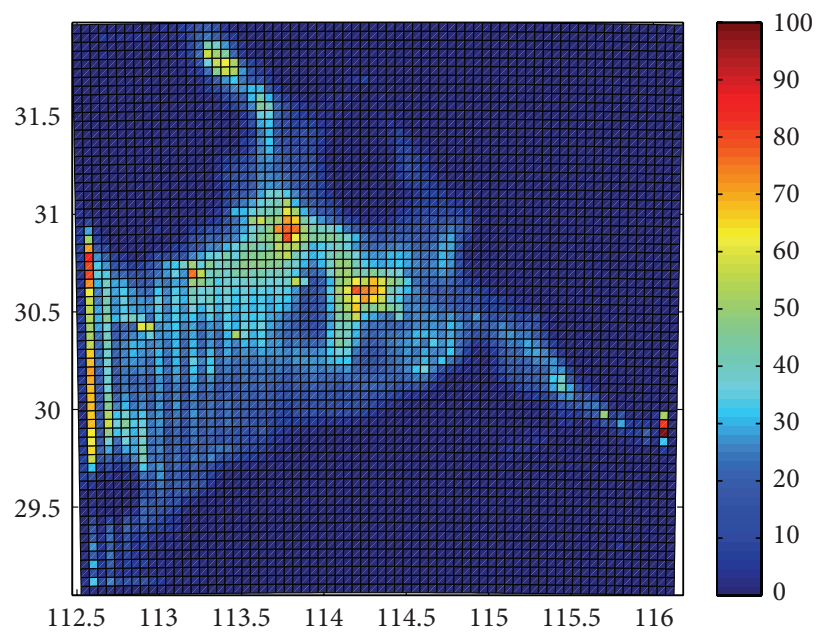

(c) Decentralized scenario

FIGURE 8: Spatial distribution of precipitation under different scenarios (unit: $\mathrm{mm}$ ). 
impact on regional climate by changing the underlying surface. As an important place for human settlements, urban development should be evaluated form both environmental effects and its impact on human welfare when pursuing economic efficiency. Therefore, not only economic efficiency differences but also the environmental effects of urban development and its impact on human living conditions should be considered to choose a rational urban land expansion pattern. Especially in the context of global climate change, studying impacts of underlying surface on regional climate change has important guiding significance for selection of rational urbanization patterns.

China is currently in the period of rapid urbanization. In the coming period, China will maintain a high speed of urbanization. Therefore, rational choice of urbanization model has a special significance for China. Urban agglomeration has gradually become the main form of urbanization in China. Wuhan Metropolitan is an important urban agglomeration after the rise of the central China following the Yangtze River Delta, Pearl River Delta, and the Bohai Sea Rim in China. It is also the first batch of pilot area of the "resources-saving and environment-friendly" society construction in China. This paper took Wuhan Metropolitan as the case area, based on using Partitioned \& Asynchronous Cellular Automata Model to carry out urban land expansion scenario simulation under different urbanization patterns. And then, the impact of the underlying surface changes on regional temperature and precipitation in different urbanization scenarios are simulated and analyzed by WRF model. Results show the following.

(1) Under different urban land expansion scenarios, warming effect of urban land expansion has significant differences. Warming effect of centralized urbanization is the most significant, far higher than the decentralized urbanization scenario's warming effect. Warming effect of decentralized urban land expansion scenario is much weaker than the other two scenarios.

(2) Under different urban land expansion scenarios, the spatial distribution of warming effect also has significant differences. The warming effect of centralized urban land expansion is widest and strongest in three urban land expansion scenarios. The warming effect of decentralized urban land expansion scenario is on the opposite.

(3) The different effects of different urban land expansion scenarios on regional average precipitation are not very significant. Overall, the regional precipitation is largest under decentralized urban land expansion scenario, slightly higher than the other two scenarios. On the opposite, the regional precipitation under centralized urban land expansion scenario is smallest. The precipitations under difference scenarios are comparatively consistent in time distribution.

(4) Under different urban land expansion scenarios, spatial distribution of precipitation has significant differences. The spatial distribution of precipitation is more concentrated in centralized urban land expansion scenario, and presents a wider scope and greater intensity of rainfall center. The distribution of precipitation is more dispersed and the rainfall centers are still dispersed under decentralized urban land expansion.

(5) Decentralized urban land expansion pattern is the suitable urbanization pattern for mitigating the climate change. Under decentralized urban land expansion scenario, underlying surface change has little effect on climate change, and showing a uniform distribution pattern in space. In the centralized urban land expansion scenario, there is a greater impact on climate change, and the distribution is more concentrated in the space.

By analyzing effects of urban land expansion on regional temperature and precipitation in different urban land expansion patterns, it can be concluded that decentralized urban land expansion pattern is rational in "resources-saving and environment-friendly" society construction. In further studies, we need to discuss in-depth that the spatial and temporal laws of regional underlying surface change on other parameters affects the ecological environment and its effect on the human temporal welfare under different urban land expansion scenarios.

\section{Conflict of Interests}

The authors declare that there is no conflict of interests regarding the publication of this paper.

\section{Acknowledgments}

This research was financially supported by the National Key Program for Developing Basic Science of China (Grant no. 2010CB950904), the National Science Foundation of China (Grant no. 41101098), the Ministry of Education Research of Social Sciences Youth funded Projects (Grant no. 10YJC790121), and the National Department Public Benefit Research Foundation of the Ministry of Land and Resources of China (Grant no. 201311001-5).

\section{References}

[1] G. Churkina, "Modeling the carbon cycle of urban systems," Ecological Modelling, vol. 216, no. 2, pp. 107-113, 2008.

[2] R. Bornstein and Q. Lin, "Urban heat islands and summertime convective thunderstorms in Atlanta: three case studies," Atmospheric Environment, vol. 34, no. 3, pp. 507-516, 2000.

[3] R. A. Pielke Sr., G. Marland, R. A. Betts et al., "The influence of land-use change and landscape dynamics on the climate system: relevance to climate-change policy beyond the radiative effect of greenhouse gases," Philosophical Transactions of the Royal Society A, vol. 360, no. 1797, pp. 1705-1719, 2002.

[4] M. Lei, D. Niyogi, C. Kishtawal et al., "Effect of explicit urban land surface representation on the simulation of the 26 July 2005 heavy rain event over Mumbai, India," Atmospheric Chemistry and Physics, vol. 8, no. 20, pp. 5975-5995, 2008. 
[5] X. Deng, C. Zhao, and H. Yan, "Systematic modeling of impacts of land use and land cover changes on regional climate: a review," Advances in Meteorology, vol. 2013, Article ID 317678, 10 pages, 2013.

[6] L. Ka and N. Edward, "An investigation of urbanization effect on urban and rural Hong Kong using a 40-year extended temperature record," Landscape and Urban Planning, vol. 114, pp. 42-52, 2013.

[7] Y. Xiong, S. Huang, F. Chen, H. Ye, C. Wang, and C. Zhu, “The impacts of rapid urbanization on the thermal environment: a remote sensing study of Guangzhou, South China," Remote Sensing, vol. 4, pp. 2033-2056, 2012.

[8] Z. Guo, S. Wang, M. Cheng, and Y. Shu, "Assess the effect of different degrees of urbanization on land surface temperature using remote sensing images," Procedia Environmental Sciences, vol. 13, pp. 935-942, 2012.

[9] C. M. Kishtawal, D. Niyogi, M. Tewari, R. A. Pielke, and J. M. Shepherd, "Urbanization signature in the observed heavy rainfall climatology over India," International Journal of Climatology, vol. 30, no. 13, pp. 1908-1916, 2010.

[10] M. Chen, W. Liu, and X. Tao, "Evolution and assessment on China's urbanization 1960-2010: under-urbanization or overurbanization?" Habitat International, vol. 38, pp. 25-33, 2013.

[11] L. Salvati, A. Sateriano, and S. Bajocco, "To grow or to sprawl? Land Cover Relationships in a Mediterranean City Region and implications for land use management," Cities, vol. 30, pp. 113121, 2013.

[12] J. Wu, G. D. Jenerette, A. Buyantuyev, and C. L. Redman, "Quantifying spatiotemporal patterns of urbanization: the case of the two fastest growing metropolitan regions in the United States," Ecological Complexity, vol. 8, no. 1, pp. 1-8, 2011.

[13] B. He, C. Ding, G. Xu, H. Liu, J. Liu, and Q. Zhang, "Surface temperature characteristics based on MODIS in Wuhan city circle," Resources and Environment, vol. 19, no. 12, pp. 1379-1385, 2010.

[14] J. Liu, M. Liu, and D. Zhuang, "Spatial pattern analysis of China's recent land-use change," Chinese Science, vol. 32, no. 12, pp. 10311040, 2002.

[15] X. Li, A. G. O. Yeh, and X. Liu, Geographical Simulation Systems: Cellular Automata and Multi-Agent Systems, Science Press, Beijing, China, 2007.

[16] X. Ke and F. Bian, "A partitioned \& asynchronous CA based on spatial data mining," Journal of Image and Graphics, vol. 15, no. 6, pp. 921-930, 2010.

[17] X. Ke, A partitioned \& asynchronous CA model and its sensitivity to scales [Ph.D. dissertation], Wuhan University, 2009. 

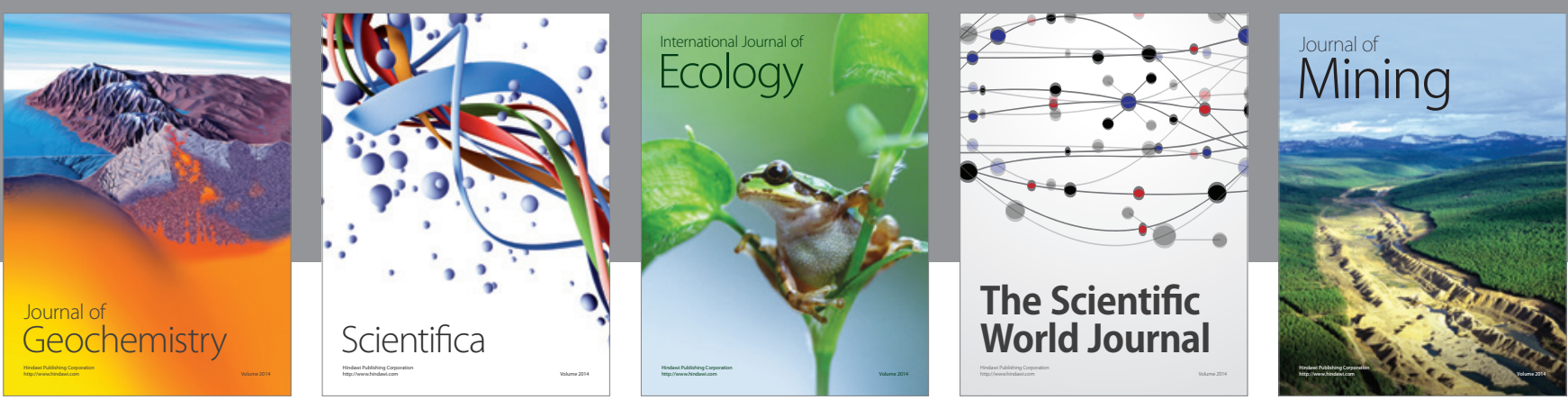

The Scientific World Journal
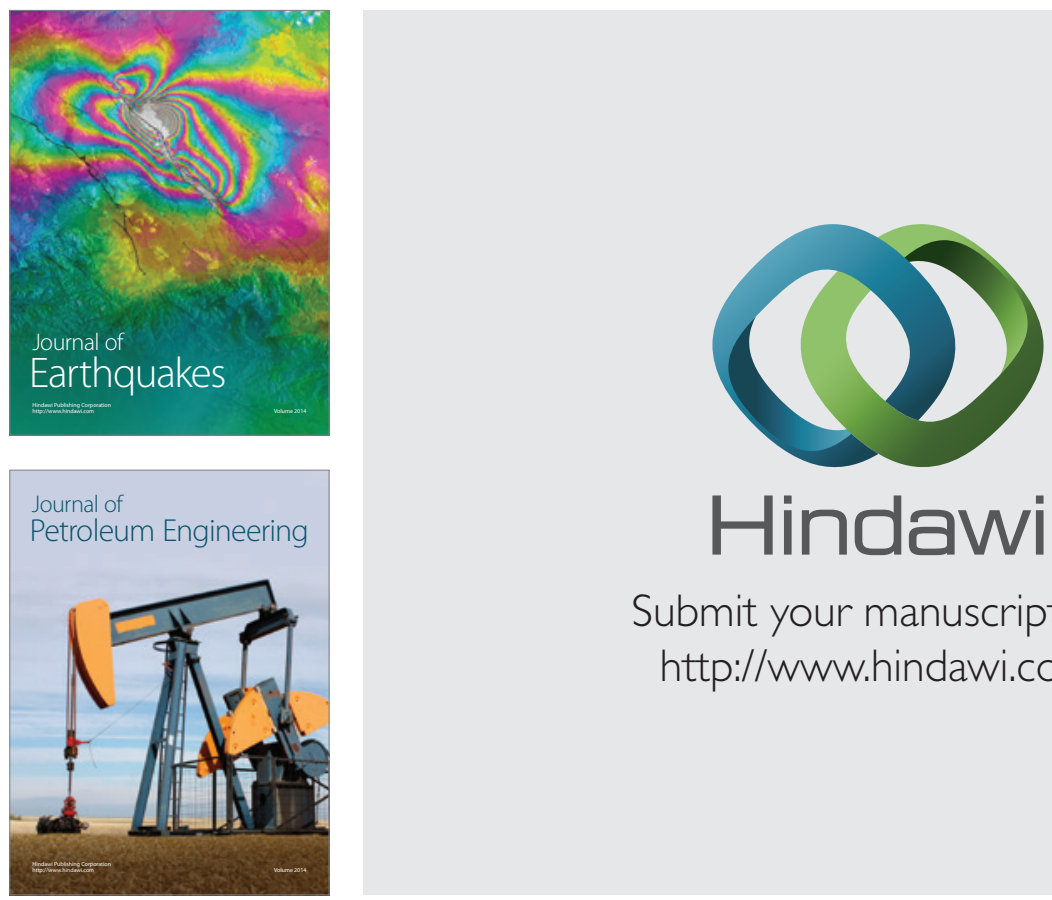

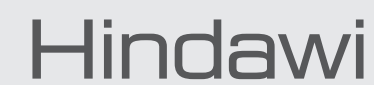

Submit your manuscripts at

http://www.hindawi.com
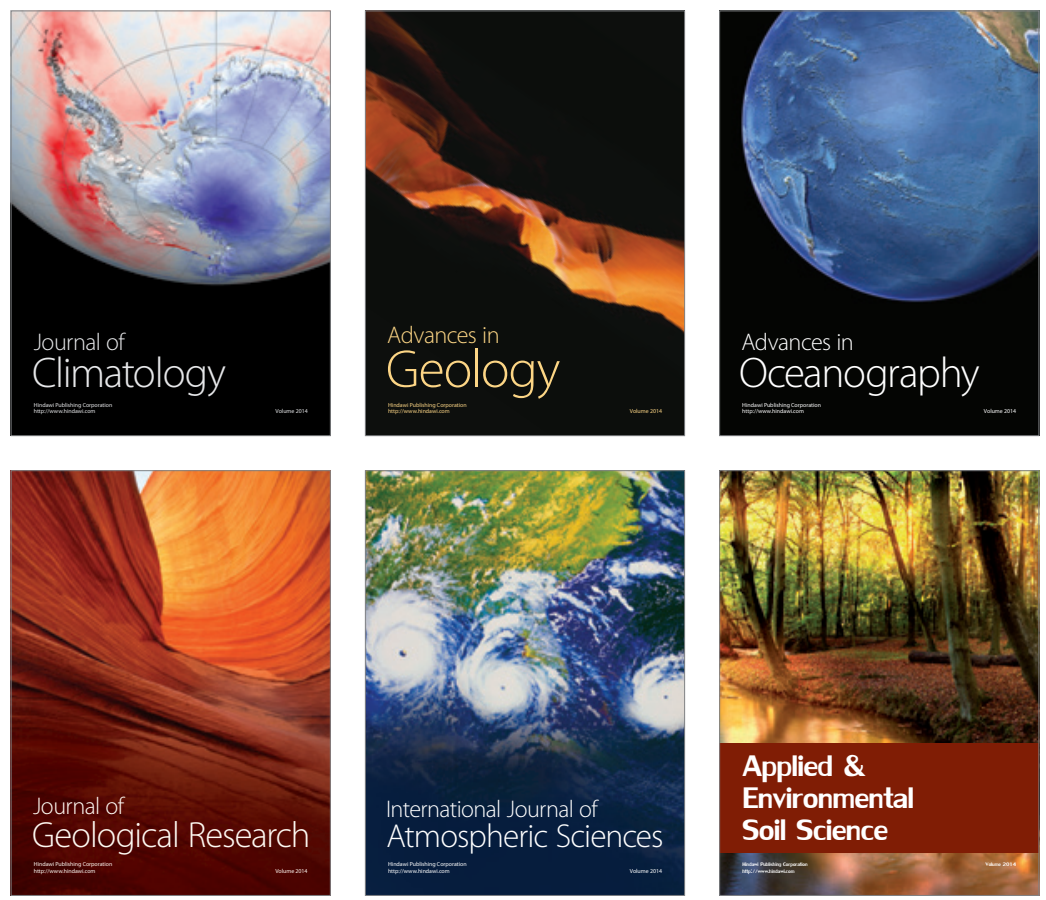
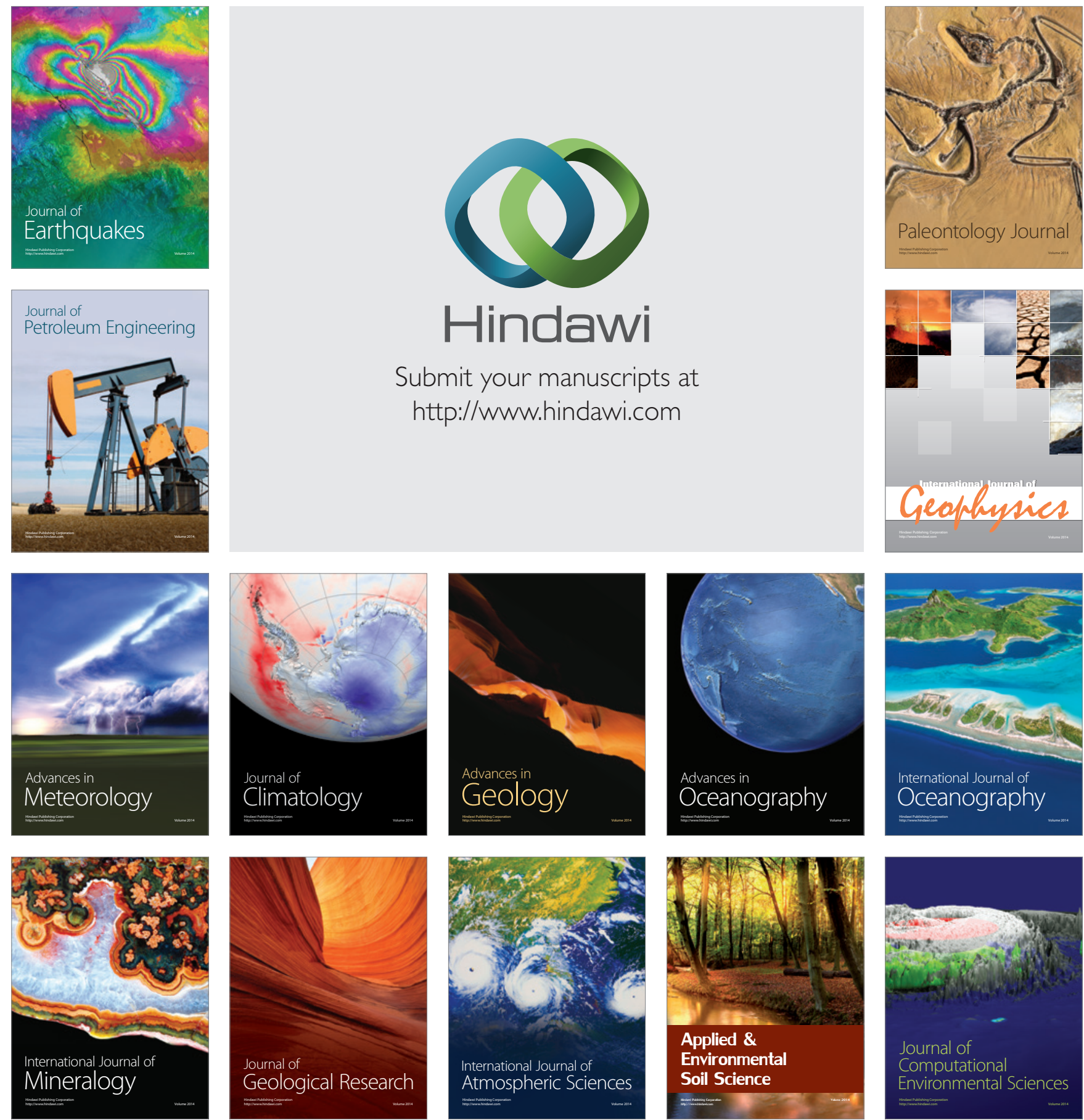